\title{
Efectos de la Clonidina sobre el Bochorno y otros Síntomas Asociados en la Mujer Menopáusica
}

\author{
Dres.: Jairo de la Cruz Segura*, Hernando Alberto Santos y Juan Pablo Suso Ayerbe
}

\section{ANTECEDENTES Y JUSTIFICACION}

El bochorno es el síntoma que puede anunciar la menopausia meses o años antes de que terminen las menstruaciones en la mujer climatérica (1).

Este síntoma, más molesto que ningún otro de los que acompañan a la menopausia, hace que la mujer busque alivio terapéutico. A pesar del aumento de la longevidad en las últimas décadas, el promedio de edad para la cesación de la función ovárica ha permanecido estable aproximaramente en 51.4 años (3).

El bochorno se presenta en un 65 a $75 \%$ de las pacientes postmenopáusicas (1.2.4.), y en un 37 a $50 \%$ de las mujeres pre-menopáusicas que sufren ovariectomía bilateral (5).

Pocas áreas en la Medicina han creado tal confusión como la que concierne al sindrome menopáusico y su tratamiento.

* Profesor Asistente Escuela Militar de Medicina. Sección Gineco-Obstetricia. Hospital Militar Central.
El bochorno es descrito como una brusca sensación de calor en la cara que progresa hacia el cuello y el tórax. Esta sensación de calor dura unos dos minutos y van sequidos por escalofríos, sudoración y enrojecimiento de la piel. Algunas presentan además, aturdimiento, vértigo, cefaleas, palpitaciones y náuseas inmediatamente después del bochorno.

La resolución de estos síntomas molestos es tan brusca como el comienzo; todo el episodio ha terminado en unos pocos minutos.

A diferencia de otros síntomas de la menopausia como la desmir,eralización osea, la sequeda vaginal, la pérdida de la líbido, la dispareunia, el bochorno disminuye de frecuencia $y$ de intensidad a medida que pasan los años.

Se han descrito síntomas similares en pacientes con feocromocitoma $(6,7)$ y en pacientes con epilepsia del sistema autónomo que secretaba catecolaminas durante una crisis (8).

La similitud entre los trastornos por exceso de catecolaminas y el bochorno menopáusico, ha hecho que los investi- 
gadores buscaran una explicación basada en alguna alteración del metabolismo de las catecolaminas $(9,10)$.

El que el estrógeno sustitutivo alivie los episodios del bochorno depende de una desaparición del estrógeno $(1,3$, $11,12)$.

A pesar de que la terapia con estrógenos es efectiva para prevenir estos síntomas, se ha encontrado asociado un al to riesgo en estas pacientes a desarrollar cáncer de-endometrio $(11,13,14)$, cáncer del seno (14, 15), litiasis biliar (16), e hipertensión $(3,17)$; por lo tanto, la terapia estrogénica presenta contraindicaciones como son las neoplasias dependientes de estrógenos en seno y útero, sangrados genitales anormales sin diagnóstico, historia de enfermedad vascular periférica, enfermedad hepática aguda, diabetes mellitus, miomatosis uterina, porfiria e hipertensión arterial severa (3).

En atención a estos hallazgos se han buscado tratamientos alternativos para evitar estos posibles riesgos. Estos tratamientos incluyen: placebo, tranquilizantes $y$ sedantes, propanolol y progestinas $(10,18,19,20)$.

La clonidina usada en el tratamiento de la hipertensión arterial ha sido informada como un medicamento efectivo para reducir la frecuencia del bochorno, aunque esto no es afirmado por todos los autores $(2,10,21,22)$. Este agente puede ser de particular beneficio en pacientes hipertensas con bochornos, en las que la elevación de la presión puede ser estimulada por los estrógenos (2).

\section{HIPOTESIS}

La similitud entre bochorno menopáusico y síntomas experimentados por personas con feocromocitoma, sugiere que la alteración del sistema nervioso autónomo desempeña un papel causal en la fisiopatología del bochorno (9).

Algunos autores han logrado disminuir los bochornos menopáusicos administrando un agonista alfa-adrenérgico.

La clonidina es un agente agonista alfaadrenérgico que estimula selectivamente los receptores alfa-adrenérgicos post-sinápticos en el centro vasomotor depresor en la médula (2); además, también puede influenciar estructuras suprabulbares tales como receptores alfa-adrenérgicos en el hipotálamo.

Es posible que la administración de la clonidina pueda disminuir la frecuencia e intensidad de los bochornos menopáusicos y otros sintomas asociados.

\section{OBJETIVO}

Administrar clonidina como tratamiento médico a las pacientes que presenten bochornos menopáusicos y síntomas asociados, ya sea por menopausia espontánea o quirúrgica.

\section{PROPOSITOS}

A. Probar la eficacia de la clonidina en el control de los bochornos menopáusicos.

B. Establecer un tratamiento alternativo para el control de los bochornos menopáusicos.

C. Valorar el efecto de la clonidina en pacientes hipertensas con bochornos $y$ síntomas menopáusicos asociados.

\section{UNIVERSO Y MUESTRA}

El presente trabajo se realizó en un grupo de cuarenta y cinco (45) pacientes con menopausia espontánea o provocada quirúrgicamente, que refirieron bochornos $y$ síntomas asociados y que ingresa- 
ron en la consulta externa de ginecología, en el Hospital Militar Central.

\section{TECNICAS Y PROCEDIMIENTOS}

A.Se incluyeron en el trabajo sólo las pacientes con menopausia espontánea o provocada quirúrqicamente, que refirieron bochornos y síntomas asociados.

B. Se valoró cualitativamente la intensidad y frecuencia de los síntomas referidos en el momento del ingreso al estudio.

C. Se administró a las pacientes seleccionadas, 75 microgramos (mcgrs.). de clonidina cada 12 horas en forma continua durante dos semanas.

D. Se practicó un control terminadas las dos semanas de tratamiento, valorando nuevamente en forma cualitativa la intensidad y frecuencia diaria de los sintomas.

E. Se registraron los efectos colaterales ocasionados por la clonidina en las pacientes tratadas.

F. No se incluyeron en el. estudio aqueIlas pacientes que estaban recibiendo terapia alguna diferente a la administrada en este trabajo.

G.Se excluyeron las pacientes que presentaron efectos colaterales intolerables para la paciente, ocasionados por la CLONIDINA.

H. Se administró a un grupo de 8 pacientes con iguales características a las descritas para ser incluidas en el presente trabajo, un placebo durante un período similar $y$ en igual forma al de la clonidina, siendo este grupo también controlado a las dos semanas de iniciado el tratamiento. Posteriormente a este mismo grupo se les administró clonidina en la forma descrita para obtener datos comparativos.

\section{ANALISIS DE RESULTADOS}

Se sometieron a estudio cuarenta y cinco (45) pacientes de las cuales cuatro (4) no regresaron a control; otras tres (3) pacientes se excluyeron del trabajo, dos de ellas por toma irregular e inadecuada de la droga, y una (1) por haber iniciado hormonoterapia automedicada. Las restantes treinta y ocho (38) pacientes completaron el estudio, de las cuales ocho (8) recibieron inicialmente terapia con placebo y posteriormente fueron sometidas al igual que las otras treinta (30) a tratamiento con Clonidina.

El rango de edad de las pacientes estudiadas varió entre 36 y 61 años. La edad promedio fue de 49 años. El tiempo promedio de menopausia fue 2.5 años.

Entre las pacientes estudiadas veintiocho (28) (73.68\%) iniciaron espontáneamente la menopausia, y en diez (10) (28.31은 fue inducida quirúrgicamente.

De las treinta y ocho (38) pacientes, solamente siete (7) (18.42\%) pacientes tenían antecedentes de haber recibido terapia hormonal sustitutiva.

En catorce (14) (36.84\%) de las pacientes que referían bochornos y otros síntomas menopáusicos se encontró asociada hipertensión arterial.

En el Cuadro No. 1 se muestran los principales síntomas menopáusicos referidos por las pacientes siendo el bochorno (100\%) la sudoración (89.47\%) y la cefálea los más frecuentes e intensos, seguidos por las palpitaciones y el insomnio. Con menor frecuencia se presentaron las náuseas y el escalofrío. Estos resultados se evidencian más claramente en el Gráfico No. 1. 
Cuadro No. 1

FRECUENCIA E INTENSIDAD DE LOS PRINCIPALES SINTOMAS MENOPAUSICOS DE LAS PACIENTES EN ESTUDIO

\begin{tabular}{|c|c|c|c|c|c|c|c|}
\hline \multirow{3}{*}{ SINTOMAS } & \multicolumn{5}{|c|}{ No. CASOS } & \multicolumn{2}{|c|}{ INTENSIDAD } \\
\hline & \multirow[b]{2}{*}{ No. } & \multirow[b]{2}{*}{$\%$} & Severa & \multicolumn{2}{|c|}{ Moderada } & \multicolumn{2}{|c|}{ Leve } \\
\hline & & & No. $\%$ & No. & $\%$ & No. & $\%$ \\
\hline BOCHORNO & 38 & 100 & $\begin{array}{ll}33 & 86.84\end{array}$ & 5 & 13.16 & - & - \\
\hline SUDORACION & 34 & 89.47 & $23 \quad 67.64$ & 9 & 26.47 & 2 & 5.88 \\
\hline ESCALOFRIO & & 42.10 & $4 \quad 25.00$ & 6 & 37.50 & 6 & 37.50 \\
\hline CEFALEA & 29 & 76.31 & 1344.82 & 12 & 41.37 & 4 & 13.79 \\
\hline PALPITACION & 25 & 65.78 & 1144.00 & 10 & 40.00 & 4 & 16.00 \\
\hline NAUSEA & 8 & 21.05 & 1.12 .50 & 5 & 62.50 & 2 & 25.00 \\
\hline INSOMNIO & 20 & 52.63 & 1050.00 & 8 & 40.00 & 2 & 10.00 \\
\hline
\end{tabular}

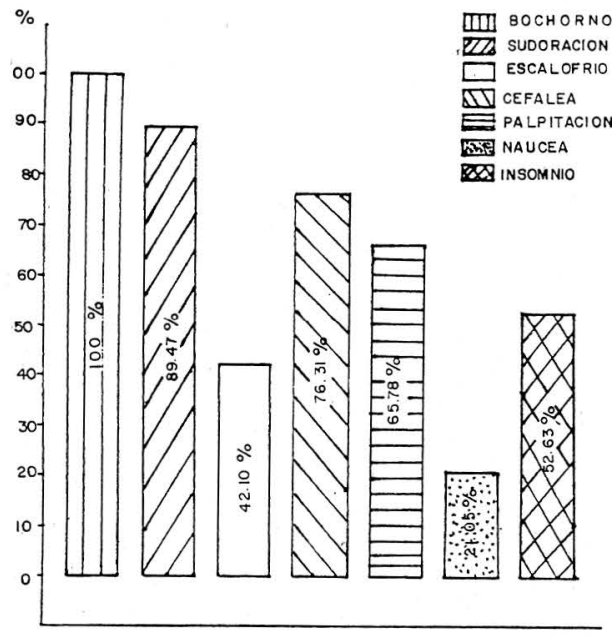

El Cuadro No. 2 muestra el tipo de las pacientes con bochorno a la administración de clonidina, encontrándose que ocurrió mejoría en el 89.48 \% de las pacientes, de las cuales el $52.64 \%$ presentaron mejoría completa y el $36.84 \%$ tuvieron mejoría parcial. Dentro de este grupo en cuatro (4) pacientes se obtuvo mejoría cuando se aumentó la dosis de 150 mcgrs. de clonidina a 225 mcgrs. de la misma. Solamente cuatro (4) (10.52\%) de las treinta y ocho (38) pacientes estudiadas no presentaron ninguna mejoría con respecto a su sintomatología inicial a pesar de haber aumentado la dosis de clonidina. El bochorno fue de intensidad severa en el $89.46 \%$ de las pacientes. No se encontró diferencia significativa en la respuesta al tratamiento 
Cuadro No. 2

\section{RESPUESTA DEL BOCHORNO MENOPAUSICO AL TRATAMIENTO CON CLONIDINA - SEGUN INTENSIDAD DEL SINTOMA}

\begin{tabular}{|c|c|c|c|c|c|c|c|c|}
\hline $\begin{array}{l}\text { RESPUESTA AL } \\
\text { rRATAMIENTO }\end{array}$ & NO & JORIA & $\begin{array}{l}\text { MEJ } \\
\text { PAR }\end{array}$ & & $\begin{array}{l}\text { MEJC } \\
\text { COMF }\end{array}$ & $\begin{array}{l}\text { RIA } \\
\text { LETA }\end{array}$ & $\mathrm{T} \quad \mathrm{O}$ & I A L \\
\hline $\begin{array}{l}\text { INTENSIDAD } \\
\text { BOCHORNO }\end{array}$ & No. & $\%$ & No. & $\%$ & No. & $\%$ & No. & $\%$ \\
\hline SEVERO & 3 & 7.89 & 13 & 34.21 & 18 & 47.36 & 34 & 89.46 \\
\hline MODERADO & 1 & 2.63 & 1 & 2.63 & 2 & 5.28 & 4 & 10.54 \\
\hline $\begin{array}{lllll}\mathrm{T} & 0 & \mathrm{~T} & \mathrm{~A} & \mathrm{~L}\end{array}$ & 4 & 10.52 & 14 & 36.84 & 20 & 52.64 & 38 & 100 \\
\hline
\end{tabular}

con clonidina, con respecto a la intensidad de los síntomas.

En el cuadro No. 3 se muestra la respuesta de las ocho (8) pacientes a las que se les administró placebo, observándose que en ninguna hubo mejoría completa, y sólo en dos (2) hubo mejoría parcial, siendo esta en ambos casos de tipo leve. En las seis (6) restantes no hubo ningún tipo de mejoría.

En el gráfico No. 2 al correlacionar los resultados de los cuadros No. 2 y 3 , apreciamos en forma clara y esquemática las diferencias significativas encontradas entre los grupos que presentaron mejoría completa y los que no presentaron ninguna mejoría.

Cuando se administró clonidina a las ocho (8) pacientes que previamente habían recibido placebo se encontró inversión en la respuesta al presentar
Gráfico No. 2

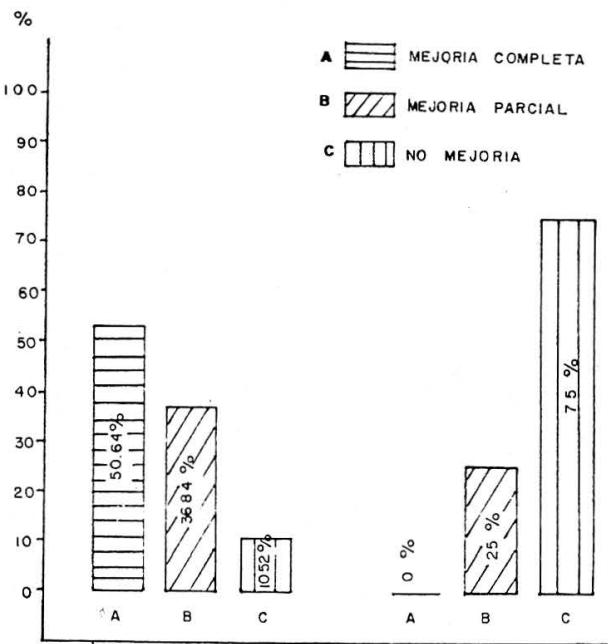


Cuadro No. 3

\section{RESPUESTA DEL BOCHORNO MENOPAUSICO AL TRATAMIENTO CON PLACEBO - SEGUN INTENSIDAD DEL SINTOMA}

\begin{tabular}{|c|c|c|c|c|c|c|c|c|}
\hline \multirow{2}{*}{$\begin{array}{l}\text { RESPUESTA AL } \\
\text { TRATAMIENTO } \\
\text { INTENSIDAD } \\
\text { BOCHORNO }\end{array}$} & \multicolumn{2}{|c|}{ NO MEJORIA } & \multicolumn{2}{|c|}{$\begin{array}{l}\text { MEJORIA } \\
\text { PARCIAL }\end{array}$} & \multicolumn{2}{|c|}{$\begin{array}{l}\text { MEJORIA } \\
\text { COMPLETA }\end{array}$} & \multicolumn{2}{|c|}{ 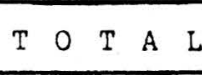 } \\
\hline & No. & $\%$ & No & $\%$ & No. & $\%$ & No. & $\%$ \\
\hline SEVERO & 6 & 75.00 & 2 & 25.00 & - & - & 8 & 100 \\
\hline MODERADO & - & - & - & - & - & - & - & - \\
\hline TOTAL & 6 & 75.00 & 2 & 25.00 & - & - & 8 & 100 \\
\hline
\end{tabular}

mejoría completa en 6 casos y persistir mejoría parcial en 2, siendo los mismos que tuvieron mejoría parcial cuando se administró placebo. Estos datos aparecen en el cuadro No. 4.

En la gráfica No. 3 se observa la correlación entre el grupo de catorce (14) pacientes hipertensas con el grupo de veinticuatro (24) pacientes normotensas, encontrándose en el primero mejoría completa en el $78.57 \%$, mejoría parcial en el $14.28 \%$ y solamente no se obtuvo ninguna mejoría en una paciente (7.14ㅇ). En el grupo de las normotensas sólo hubo mejoría completa en el $50 \%$, aunque se puede observar que el 100 \% de ellas presentaron respuesta benéfica. La totalidad de las pacientes hipertensas refirieron bochorno como un síntoma. de intensidad severa.

En el cuadro No. 5 se observa el efecto del tratamiento con clonidina sobre otros síntomas menopáusicos asociados al bochorno, encontrando una mejoría general en toda la sintomatología con mayor porcentaje para la mejoría completa en cada uno de ellos, siendo el insomnio con el $77.77 \%$ el de mejor respuesta, seguido por la sudoración con $64.7 \%$, náuseas el $66.66 \%$. La cefalea presentó mejoría en el 93.75\% aunque la mayoría de este porcentaje (56.25\%) fue en forma parcial.

Gráfico No. 3

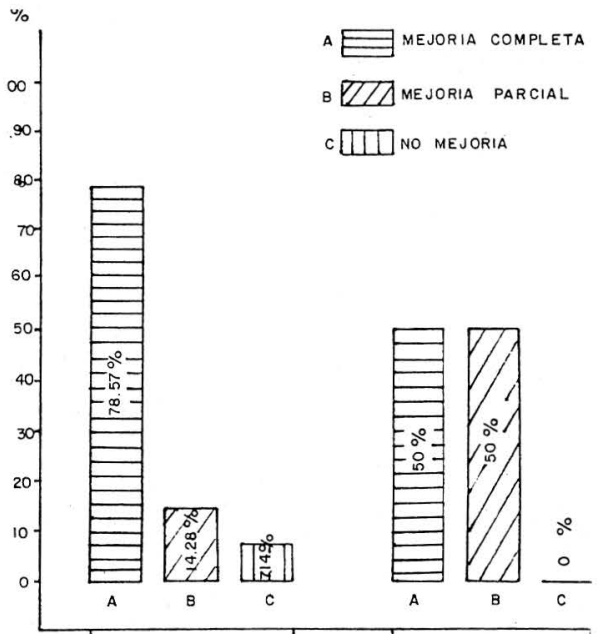


Cuadro No. 4

EFECTOS DE LA CLONIDINA SOBRE EL BOCHORNO MENOPAUSICO EN PACIENTES SIN MEJORIA TRATAMIENTO CON PI AC.FRO

\begin{tabular}{|c|c|c|c|c|c|c|c|c|}
\hline \multirow{2}{*}{$\begin{array}{l}\text { RESPUESTA AL } \\
\text { TRATAMIENTO } \\
\text { TIPO DE DROGA }\end{array}$} & \multicolumn{2}{|c|}{ NO MEJORIA } & \multicolumn{2}{|c|}{$\begin{array}{l}\text { MEJORIA } \\
\text { PARCIAL }\end{array}$} & \multicolumn{2}{|c|}{$\begin{array}{l}\text { MEJORIA } \\
\text { COMPLETA }\end{array}$} & \multicolumn{2}{|c|}{$\mathrm{T} \quad \mathrm{O} \quad \mathrm{T} \quad \mathrm{A} \quad \mathrm{I}$} \\
\hline & No. & $\%$ & No. & $\%$ & No. & $\%$ & No. & $\%$ \\
\hline PLACEBO & 6 & 75.00 & 2 & 25.00 & - & - & 8 & 100 \\
\hline CLONIDINA & - & - & 2 & 25.00 & 6 & 75.00 & 8 & 100 \\
\hline
\end{tabular}

Cuadro No. 5

EFECTOS DEL TRATAMIENTO CON CLONIDINA SOBRE OTROS SINTOMAS ASOCIADOS AL BOCHORNO

\begin{tabular}{|c|c|c|c|c|c|c|c|c|}
\hline \multirow{2}{*}{$\begin{array}{l}\text { RESPUESTA AL } \\
\text { TRATAMIENTO } \\
\text { TIPO DE } \\
\text { SINTOMA }\end{array}$} & \multicolumn{2}{|c|}{$\begin{array}{c}\text { NO } \\
\text { MEJORIA }\end{array}$} & \multicolumn{2}{|c|}{$\begin{array}{l}\text { MEJORIA } \\
\text { PARCIAL }\end{array}$} & \multicolumn{2}{|c|}{$\begin{array}{l}\text { MEJORIA } \\
\text { COMPLETA }\end{array}$} & \multicolumn{2}{|c|}{ TOTAL } \\
\hline & No & $\%$ & No. & $\%$ & No. & $\%$ & No. & $\%$ \\
\hline SUDORACION & & 11.76 & 8 & 23.52 & 22 & 64.70 & 34 & 100 \\
\hline ESCALOFRIO & & 7.69 & 5 & 38.46 & 7 & 53.84 & 13 & 100 \\
\hline CEFALEA & & 6.25 & 18 & 56.25 & 12 & 37.50 & 32 & 100 \\
\hline PALPITACION & & 13.63 & 10 & 45.45 & 9 & 40.90 & 22 & 100 \\
\hline NAUSEA & & 16.66 & 1 & 16.66 & 4 & 66.66 & 6 & 100 \\
\hline INSOMNIO & & 5.55 & 3 & 16.66 & 14 & 77.77 & 18 & 100 \\
\hline
\end{tabular}


En el cuadro No. 6 se presentan los efectos colaterales ocasionados por la clonidina en las pacientes estudiadas, aclarando que ninguna de ellas fue intolerable en algún momento que hubiese requerido suspender el tratamiento ordenado. En orden de frecuencia fueron Ios siguientes: resequedad de mucosa oral y somnolencia en 34.21\%: mareo en 15.78\%; adinamia en $7.89 \%$; palpitaciones y epigastralgia en $2.63 \%$ obteniéndose como resultado que el $68.42 \%$ de las pacientes que recibieron terapia con clonidina presentaron algún síntoma colateral, mientras que el $31.57 \%$ restante no refirió molestia alguna ocasionada por la droga.

Cuadro No. 6

\section{EFECTOS COLATERALES POR LA CLONIDINA EN LAS PACIENTES ESTUDIADAS}

\begin{tabular}{||l|c|c|}
\hline $\begin{array}{l}\text { EFECTOS } \\
\text { COLATERALES }\end{array}$ & $\begin{array}{l}\text { CASOS } \\
\text { No. }\end{array}$ & $\%$ \\
\hline NINGUNO & 12 & 31.57 \\
\hline RESEQUEDAD MUOSA ORAL & 13 & 34.21 \\
\hline MAPEO & 6 & 15.78 \\
\hline SOMNOLENCIA & 13 & 34.21 \\
\hline PALPITACION & 1 & 2.63 \\
\hline ADINAMIA & 3 & 7.89 \\
\hline EPIGASTRALGIA & 1 & 2.63 \\
\hline
\end{tabular}

\section{CONCLUSIONES}

El bajo porcentaje de pacientes con terapia hormonal sustitutiva puede ser el reflejo del temor por parte del cuerpo. médico de ocasionar efectos indeseables con estas drogas.
El alto índice de pacientes menopáusicas sin ningún tipo de terapia para sus síntomas indica la necesidad de encontrar un tratamiento eficaz e inocuo para este tipo de patología.

Los principales síntomas menopáusicos que obligan a consultar a la paciente son bochorno, sudoración y cefalea.

La clonidina es eficaz para el control del bochorno menopáusico en el $90 \%$ de los casos.

El bochorno es el síntoma más molesto para la paciente menopaúsica.

El efecto benéfico de la clonidina sobre los síntomas menopáusicos es independiente de la intensidad de los mismos.

La administración de placebo no es eficaz para el control de la sintomatología menopáusica.

La mejoría con clonidina de las pacientes que previamente no mejoraron con placebo sugiere la presencia de un factor orgánico para la explicación de los síntomas menopáusicos.

Las pacientes menopáusicas con hipertensión asociada sometidas a terapia con clonidina presentan en un porcentaje significativamente mayor desaparición completa de sus síntomas en comparación con las pacientes normotensas.

La terapia con clonidina es eficaz para el control de otros síntomas menopáusicos asociados al bochorno en un 65 a $80 \%$ de los casos.

Un alto porcentaje de las pacientes presentan efectos colaterales tolerables a la administración de clonidina.

La clonidina administrada en dosis bajas no ocasiona efectos colaterales intolerables por la paciente. 
La clonidina es un medicamento eficaz $y$ alternativo para el tratamiento del bochorno y otros síntomas asociados en las mujeres menopáusicas.

\section{RECOMENDACIONES}

Incluir la clonidina como droga alternativa para el tratamiento del bochorno y otros síntomas asociados en las mujeres menopáusicas.

Prestar mayor atención a las pacientes que refieran síntomas menopáusicos y ofrecerles un tratamiento efectivo.

Hacer de este trabajo un estudio prospectivo que incluya un mayor número de casos para obtener una muestra aún más representativa.

\section{RESUMEN}

Se someten a terapia treinta y ocho (38) pacientes con 150 mcgrs. de clonidina al día durante dos semanas, de las

\section{REFERENCIAS}

1. BATES G.W., Naturaleza del Bochorno. Clin Obstet. Ginecol. Marzo; 24 (1): 237-247, 1981.

2. LAVFER L.R. et al. Effect of Clonidine on Hot Flashes in Postmenopausal Women. Obst. Gynecol. Nov.; 60 (5); 583 $585,1982$.

3. HAMMOND CH. B. and MAXSON W.S. Current Status of Strogen Therapy for the Menopause. Fertil. Steril. Jan.; 37, (1) $5-25,1982$.

4. MULLEY G. and MITCHEL J.R.A. Menopausal Flushing. Does Oestrogen Therapy Make Sense. Lancet 1: 1.397, 1976. cuales ocho recibieron terapia inicial con placebo por igual período, observándose posteriormente los cambios en la sintomatología menopáusica como respuesta a la terapia dada y los efectos colaterales de la droga.

Se establece una comparación entre los efectos observados al administrar clonidina o placebo encontrándose que se controló en un $90 \%$ de los casos el bochorno y en un 65\% a $80 \%$ los otros síntomas menopáusicos asociados con la clonidina, mientras que con el placebo no se obtuvo mejoría alguna. No se encontraron efectos colaterales intolerables por las pacientes. Las pacientes hipertensàs, con síntomas menopáusicos asociados presentaron una mayor respuesta a la clonidina.

Se concluye que la clonidina sirve como tratamiento eficaz $y$ alternativo para el tratamiento del bochorno y otros síntomas asociados en las mujeres menopáusicas.

5. AKSEL S. et. al Vasomotor Symptoms, Serum Estrogens, and Gonadotropin Levels in Surgical Menopause. Am. J. Obstet. Gynecol. 126: 165, 1976.

6. HICKLER R.B. and THORN G.W. Pheocromocytoma, Harrison's. Principals of Internal Medicine. New York, Mc GrawHill. 557-563, 1977.

7. STUDD J. et al. The Climateric. Clin. Obstet. Gynecol. Dic.; 20 (4): 3, 1977.

8. METZ S.A. et al. Automic Epilepsy: Clonidine Blockade of Paroxysmal Catecholamine Release in Flushing. Ann. Inter. Med. 88: 189, 1978.

9. STURDEE D.W. et al. Physiological Aspect of Menopausal Hot Flush. BR. Med. J. 2: 79, 1978. 
10. CLAYDEN J.R., BELL J.W. and POLLARD P., Menopausal Flushing Double Blind Trial of a Non-Hormonal Medication. BR. Med. J. 1: 409, 1974.

11. CAMBELL $S$. and WIFLEHEAD $M$. Estrogen Therapy and the Postmenopausal Syndrome. Clin. Obst. Gynecol. Dic.; 20 (4): 31, 1977.

12. TATARYN I.V. et al. Objetive techniques for the Assesment of Postmenopausal Hot Flushes. Obstet. Gynecol. Jan.: 57: $340,1981$.

13. LICK H. et al. Replacemente Estrogens and Endometrial Cancer. N. Engl. J. Med 300: 218, 1979.

14. HAMMOND C.B. et al. Effects on Long Term Estrogen Replacement Theraphy II Neoplasia. Am. J. Obstet. Gynecol. 133; $537,1979$.

15. ROSS R.K. et al. A Case Control Study of Menopausal Estrogen and Breat Cancer Jama. 243: 1.635. 1980.

16. BOSTON COLLABORATIVE DRUG SUR VEILLANCE PROGRAM BOSTON UNIVERSITY MEDICAL CENTER, Surgically Confirmed Gollbladder Disease, Venous Thromboembolism, and Breast
Tumor in Relation to Postmenopausal Estrogen Therapy N. Engl. J. Med. 290: 15. 1974.

17. PETITI D.B. et al. Association of History of Gollbladder Disease With Reduced Concentration of High Density Lepoprotein Cholesterol. N. Engl. J. Med. 304: 1.396, 1981.

18. COOPE J. and WILLIAMS S.A. Study of the Effectiveness of Propanolol in Menopausal Hot Flushes. Br. J. Obstet. Gynecol. 85: 472, 1978.

19. MORRISON J.C. et al. The use of Medroxyprogesterone Acetate for Relief of Climateric Symptoms. BR. J. Obstet. Gynecol. 138: 99, 1980.

20. BULLOCK H.L. et al. Use Medroxyprogesterones Acetate to Prevent Menopausal Symptoms, Obstet. Gynecol 46: 165. 1975.

21. METZ S.A. et al. Suppression of Plasma Catecholamines and Flushing By Clonidine in Man. J. Clin. Endocrinol. Metab. 46: $83,1978$.

22. CLAYDEN J.R. Effect of Clonidine on Menopausal Flushing. Lancet. 2: 1, 361, 1972. 